\title{
Prolonged Intake of Juice (Citrus Unshiu) Reinforced with $\beta$-Crypthoxanthin Has an Effect on Circulating Bone Biochemical Markers in Normal Individuals
}

\author{
Masayoshi Yamaguchi, ${ }^{*, a}$ Aki Igarashi, ${ }^{a}$ Satoshi Uchiyama, ${ }^{a}$ Seiichi Morita, ${ }^{b}$ \\ Kuniaki Sugawara, ${ }^{b}$ and Takashi Sumida ${ }^{b}$
}

${ }^{a}$ Laboratory of Endocrinology and Molecular Metabolism, Graduate School of Nutritional Sciences, University of Shizuoka, 52-1 Yada, Shizuoka 422-8526, Japan and ${ }^{b}$ Research \& Development, Ehime Beverage, Inc., 478 Anjyoji-machi, Matsuyama 791-8603, Japan

(Received July 6, 2004; Accepted July 12, 2004)

\begin{abstract}
A change in circulating biochemical markers of bone metabolism in normal individuals with the intake of juice prepared from Satsuma mandarin (Citrus unshiu MARC.) containing $\beta$-crypthoxanthin was investigated. Twentyone volunteers (ten males and eleven females) were divided into two groups of ten volunteers (five males and five females) and eleven volunteers (five males and six females), and each group was given sequentially juice (192 ml) containing two different contents of $\beta$-crypthoxanthin once a day for 28 or 56 days as follows: either regular juice with naturally occurring $802 \mu \mathrm{g} \beta$-cryptoxanthin/100 ml or a reinforced juice containing $1500 \mu \mathrm{g} \beta$-cryptoxanthin/ $100 \mathrm{ml}$. As serum bone markers, bone-specific alkaline phosphatase, $\gamma$-carboxylated osteocalcin, bone tartrate-resistant acid phosphatase (TRAP), and N-telopeptide of type I collagen were assayed. The intake of regular juice for 28 or 56 days caused a significant increase in $\gamma$-carboxylated osteocalcin, a marker of bone formation, and the intake for 56 days produced a significant decrease in serum bone TRAP activity. Moreover, intake of the $\beta$-cryptoxanthin reinforced juice for 28 or 56 days caused a significant increase in serum $\beta$-carboxylated osteocalcin concentration and a corresponding decrease in serum bone TRAP activity and N-telopeptide of type I collagen, a marker of bone resorption. This study suggests that the intake of $\beta$-cryptoxanthin reinforced juice has a stimulatory effect on bone formation and an inhibitory effect on bone resorption in normal individuals.
\end{abstract}

Key words - $\beta$-cryptoxanthin, bone alkaline phosphatase, $\gamma$-carboxylated osteocalcin, bone tartrate-resistant acid phosphatase, N-telopeptide of type I collagen, osteoporosis

\section{INTRODUCTION}

Bone loss with aging induces osteoporosis, which is widely recognized as a major public health problem. A decrease in bone mass leads to bone fracture. Bone loss may be due to decreased bone formation and increased bone resorption. Pharmacologic and nutritional supplements can prevent bone loss caused by increasing age. ${ }^{1)}$ Food chemical factors may help to prevent bone loss with increasing age. Recent studies have shown that isoflavones (including genistein and daidzein), which are contained

\footnotetext{
*To whom correspondence should be addressed: Laboratory of Endocrinology and Molecular Metabolism, Graduate School of Nutritional Sciences, University of Shizuoka, 52-1 Yada, Shizuoka 422-8526, Japan. Tel. \& Fax: +81-54-264-5580; Email: yamaguch@u-shizuoka-ken.ac.jp
}

in soybeans, ${ }^{2-4)}$ and menaquinone-7, an analogue of vitamin $\mathrm{K}_{2}$ which is abundant in fermented soybeans, ${ }^{5-7)}$ have a stimulatory effect on osteoblastic bone formation and an inhibitory effect on osteoclastic bone resorption in vitro, thereby increasing bone mass. ${ }^{8,9)}$

Carotenoids are present in fruit and vegetables; the effect of carotenoids on bone metabolism, however, has not yet been clarified. More recently, it has been shown that $\beta$-cryptoxanthin has a unique anabolic effect on bone metabolism in vitro and in vivo. ${ }^{10-14)}$ Lutein, lycopene, and $\beta$-carotene, which are carotenoids, do not have an effect on bone calcification in vitro. ${ }^{10,11)} \beta$-Cryptoxnthin has a direct stimulatory effect on osteoblastic bone formation and an inhibitory effect on osteoclastic bone resorption in vitro. ${ }^{10-12)} \beta$-Cryptoxanthin is greatly present in Satsuma mandarin (Citrus unshiu MARC.). $\beta$-Cryp- 
toxanthin may have a role in bone health and may be important in the prevention of bone loss with increasing age.

The oral administration of $\beta$-cryptoxanthin, which was isolated from Satsuma mandarin, has been shown to induce an anabolic effect on bone components in young and aged rats in vivo, ${ }^{13,14)}$ suggesting that the intake of $\beta$-cryptoxanthin can prevent bone loss with aging.

This study was undertaken to determine whether the intake of juice (Citrus unshiu) containing $\beta$-cryptoxanthin has an effect on bone metabolism in normal individuals. We prepared regular juice and the $\beta$-cryptoxanthin reinforced juice. We found that the prolonged intake of a regular juice and a $\beta$-cryptoxanthin reinforced juice causes an alteration in serum biochemical markers of bone metabolism in normal individuals.

\section{MATERIALS AND METHODS}

Materials — Juice prepared from Satsuma mandarin (Citrus unshiu MARC.) was supplied by Ehime Beverage, Inc. (Matsuyama, Japan), in which $\beta$-cryptoxanthin is present in a volume of $802 \mu \mathrm{g}$ per $100 \mathrm{ml}$ of juice. The reinforced juice with increased $\beta$-cryptoxanthin content was made with a $\beta$-cryptoxanthin supplement isolated from Satsuma mandarin. The content of $\beta$-cryptoxanthin in the reinforced juice was $1500 \mu \mathrm{g}$ per $100 \mathrm{ml}$ of juice.

Experimental Procedures — Twenty-one adults, aged 23-47 years (ten males and eleven females), who were judged to be healthy with no abnormal liver and kidney functions as assessed by standard biochemical data, were enrolled as volunteers in this study. Informed consent was obtained from all of them. The intake of other foods with an abundance of $\beta$-cryptoxanthin was prohibited during the period of the experiment. The period for washout or intake of each type of juice was designed to be 7 days, and 28 or 56 days, respectively. The twenty-one volunteers were divided into two groups of either ten volunteers (five males and females) or eleven volunteers (five males and six females). Each group was given sequentially, once a day for 28 or 56 days, $192 \mathrm{ml}$ of juice containing one of two different contents: either 1540 or $2880 \mu \mathrm{g}$ per $192 \mathrm{ml}$ of juice. The intake of juice was performed between 10 a.m. and 12 a.m. Blood samples were collected from each at 3 p.m. and 5 p.m. on the day prior to intake (control), and at 28 and 56 days after start of the intake. Serum samples were obtained by centrifugation ( $2500 \mathrm{rpm}$ for $5 \mathrm{~min}$ ) between 30 and $60 \mathrm{~min}$ after blood collections, and were then stored at $-20^{\circ} \mathrm{C}$ until being assayed.

Analytical Procedures - Serum calcium and inorganic phosphorus concentrations were determined using a kit (Wako Pure Chemicals, Osaka, Japan). The serum $\gamma$-carboxylated osteocalcin concentration was assayed using a Gla type Osteocalcin (Gla-OC) EIA kit (Takara Shuzou, Shiga, Japan). ${ }^{15)}$ Serum bone-specific alkaline phosphatase activity was assayed using METRA BAP EIA kit (Quidel, San Diego, U.S.A.). ${ }^{16)}$ Serum bone tartrate-resistant acid phosphatase (TRAP) activity was assayed using a Bone TRAP Assay EIA kit (SBA Sciences, Turku, Finland). ${ }^{17)}$ Serum bone N-telopeptide of type I collagen was measured using OSTEOMARK NTx Serum EIA kit (Mochida Pharmaceutical Co., Ltd., Tokyo, Japan). ${ }^{18)}$

Statistical Analysis — Differences in values before and after the intake of each juice was estimated using Student's $t$-test. A paired $t$-test was used for the difference in values before and after the intake of each juice or between the two groups after each intake of period. $p$-Values less than 0.05 were considered statistically significant.

\section{RESULTS}

The change in serum biochemical markers of bone metabolism in normal individuals following the start of intake of regular juice or of the $\beta$-cryptoxanthin reinforced juice prepared from Satuma mandarin (Citrus unshiu MARC.) was examined. The period of intake for each type juice was designed to be 28 or 56 days.

Serum calcium and inorganic phosphorus concentrations were not significantly changed by the intake of regular juice for 28 or 56 days (Figs. 1 and 2 ). However, the intake of the $\beta$-cryptoxanthin reinforced juice for 28 or 56 days caused a significant decrease in serum calcium concentration (Fig. 1), and serum inorganic phosphorus concentration was significantly lowered by the intake for 56 days (Fig. 2) compared with the value obtained before intake.

Serum bone-specific alkaline phosphatase activity was not significantly changed by the intake of regular juice or the $\beta$-cryptoxanthin reinforced juice for 28 or 56 days (Fig. 3).

The serum $\gamma$-carboxylated osteocalcin concen- 


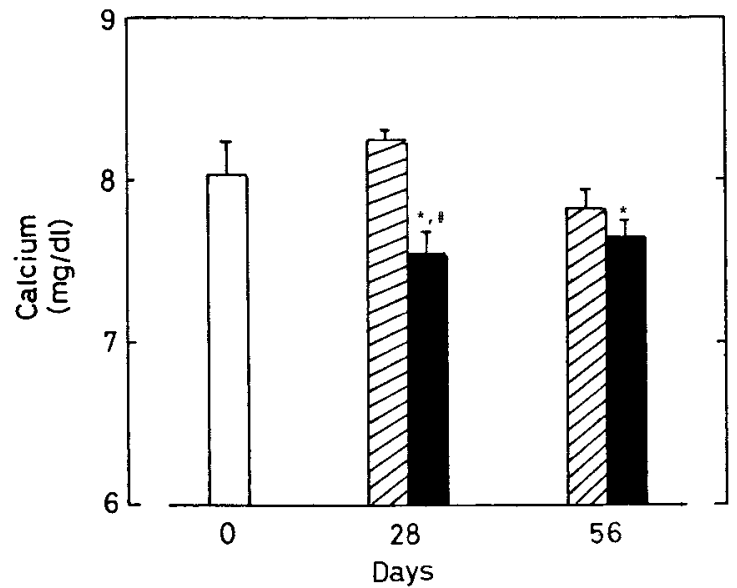

Fig. 1. Change in Serum Calcium Concentration Following Intake of Juice Containing $\beta$-Cryptoxanthin

Juice from Satsuma mandarin containing $\beta$-cryptoxanthin was given to volunteers for 28 or 56 days. Twenty-one persons (before intake) were divided into two groups ten persons for the intake of regular juice and eleven persons for the reinforced $\beta$-cryptoxanthin juice. Each value is the mean \pm SEM of ten to twenty one persons. ${ }^{*} p<0.01$, compared with the value before intake. $\# p<0.01$ compared with the value obtained from regular juice intake. White bar, before intake; hatched bars, intake of regular juice; black bars, $\beta$-cryptoxanthin reinforced juice.

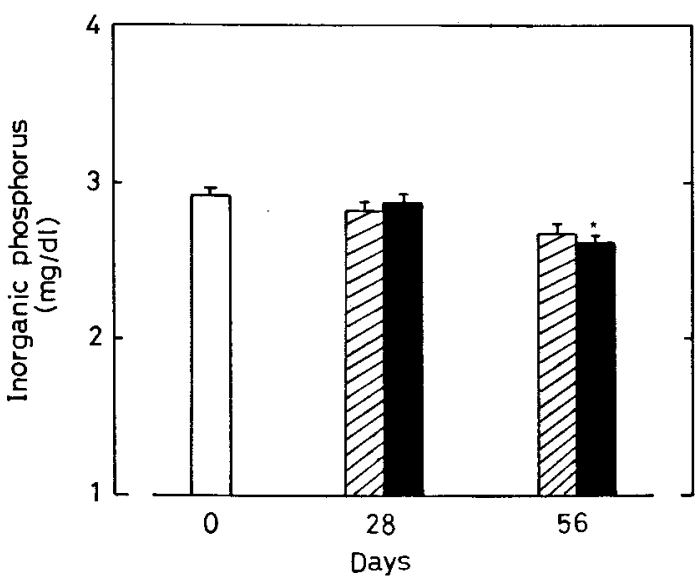

Fig. 2. Change in Serum Inorganic Phosphorus Concentration Following Intake of Juice Containing $\beta$-Cryptoxanthin

The procedure for the intake of juice containing $\beta$-cryptoxanthin described in Fig. 1. Each value is the mean \pm SEM of ten to twenty one persons. ${ }^{*} p<0.05$ compared with the value before intake. White bar, before intake; hatched bars, intake of regular juice; black bars, reinforced $\beta$-cryptoxanthin juice.

tration was significantly increased by the intake of regular juice or the reinforced $\beta$-cryptoxanthin juice for 28 or 56 days (Fig. 4). The increase in serum $\gamma$ carboxylated osteocalcin concentration was not significantly enhanced by the intake of the $\beta$-cryptoxanthin reinforced juice as compared with the value

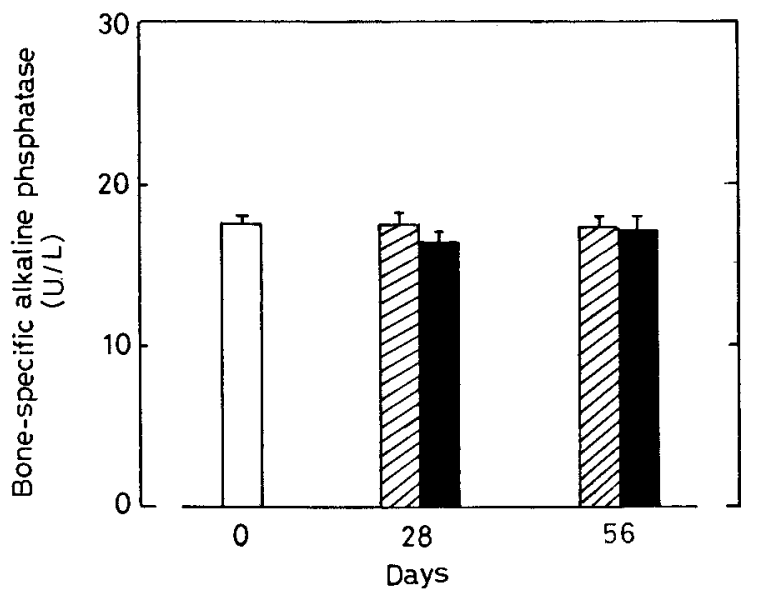

Fig. 3. Change in Serum Bone-Specific Alkaline Phosphatase Activity Following Intake of Juice Containing $\beta$ Cryptoxanthin

The procedure of the intake of juice containing $\beta$-cryptoxanthin is described in Fig. 1. Each value is the mean \pm SEM of ten to twenty-one persons. Data were not significant. White bar, before intake; hatched bars, intake of regular juice; black bars, $\beta$-cryptoxanthin reinforced juice.

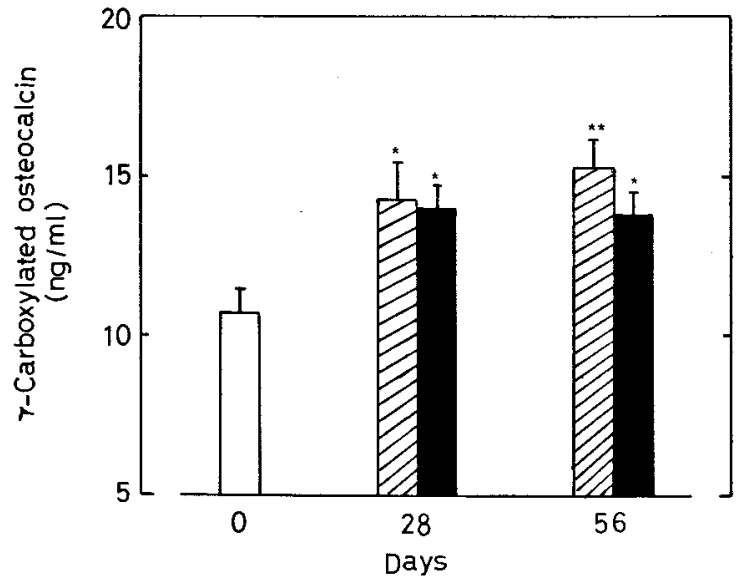

Fig. 4. Change in Serum $\gamma$-Carboxylated Osteocalcin Concentration Following Intake of Juice Containing $\beta$ Cryptoxanthin

The procedure for the intake of juice containing $\beta$-cryptoxanthin is described in Fig. 1. Each value is the mean \pm SEM of ten to twenty-one persons. ${ }^{*} p<0.05$ compared with the value before intake. $* p<0.01$ compared with the value before intake. White bar, before intake; hatched bars, intake of regular juice; black bars, $\beta$-cryptoxanthin reinforced juice.

obtained from the intake of regular juice for 28 or 56 days.

Serum bone TRAP activity was significantly decreased by the intake of regular juice for 56 days compared with the value obtained before intake (Fig. 5). Likewise, the intake of regular juice for 28 days caused a significant change in serum bone 


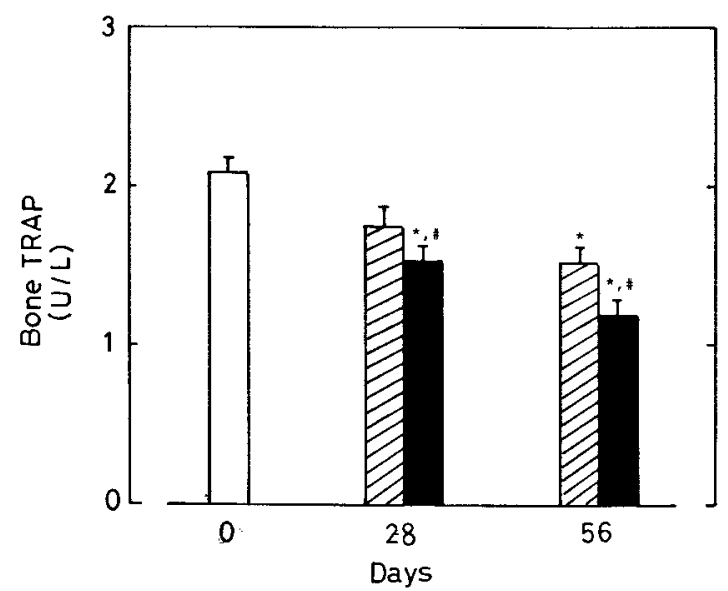

Fig. 5. Change in Serum Bone TRAP Activity Following Intake of Juice Containing $\beta$-Cryptoxanthin

The procedure for the intake of juice containing $\beta$-cryptoxanthin described in Fig. 1. Each value is the mean \pm SEM of ten to twenty one persons. $* p<0.01$ compared with the value before intake. $\# p<0.01$ compared with the value obtained from intake of regular juice. White bar, before intake; hatched bars, intake of regular juice; black bars, reinforced $\beta$-cryptoxanthin juice.

TRAP activity. Additionally, the intake of the $\beta$-cryptoxanthin reinforced juice for 28 or 56 days caused a significant decrease in serum bone TRAP activity compared with the value obtained before intake. Moreover, the intake of $\beta$-cryptoxanthin reinforced juice for 28 or 56 days had a significantly more potent reducing effect on serum bone TRAP activity compared with that of the regular juice intake.

Serum concentration of the N-telopeptide of type I collagen was significantly decreased by the intake of $\beta$-cryptoxanthin reinforced juice for 28 or 56 days, while it was not significantly changed by the intake of regular juice for 28 or 56 days compared with the value obtained before intake (Fig. 6).

\section{DISCUSSION}

Bone loss with aging induces osteoporosis, which leads to bone fracture. Bone loss may be due to decreased bone formation and increased bone resorption. Chemical factors in foods may help to prevent bone loss caused by increasing age. $\beta$-Cryptoxanthin is greatly present in Satsuma mandarin (Citrus unshiu MARC.). $\beta$-Cryptoxanthin has been demonstrated to have a stimulatory effect on bone formation and an inhibitory effect on bone resorption in vitro. ${ }^{10-12)}$ The oral administration of $\beta$-cryptoxanthin in rats induces an anabolic effect on bone com-

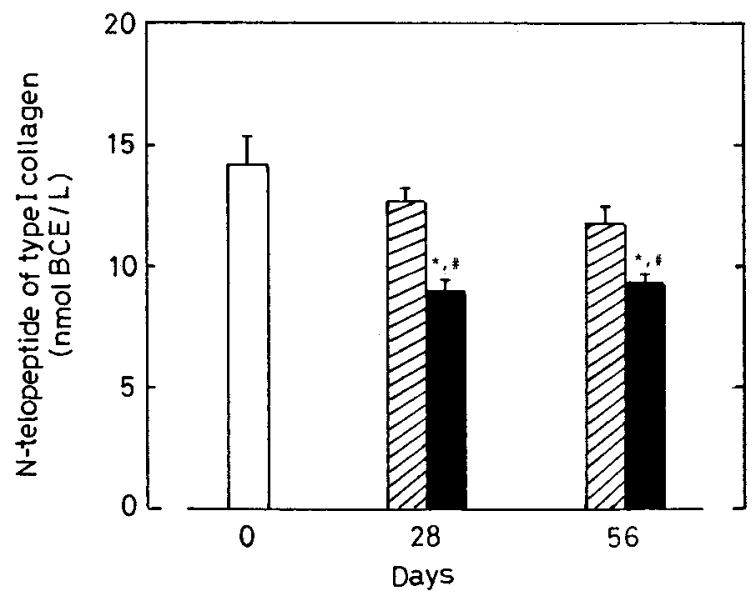

Fig. 6. Change in Serum Concentration for N-Telopeptide of Type I Collagen Following Intake of Juice Containing $\beta$-Cryptoxanthin

The procedure of the intake of juice containing $\beta$-cryptoxanthin is described in Fig. 1. Each value is the mean \pm SEM of ten to twenty-one persons. ${ }^{*} p<0.01$ compared with the value before intake. $\# p<0.01$ compared with the value obtained from the intake of regular juice. White bar, before intake; hatched bars, intake of regular juice; black bars, $\beta$ cryptoxanthin reinforced juice.

ponents in vivo, ${ }^{13,14)}$ suggesting that the intake of $\beta$ cryptoxanthin-containing juice plays a role in bone health.

This study was undertaken to determine whether the intake of $\beta$-cryptoxanthin-containing juice has an effect on specific serum biochemical markers of bone metabolism. We found that the prolonged intake of $\beta$-cryptoxanthin-containing juice causes a significant increase in serum markers of bone formation and a corresponding decrease in serum markers of bone resorption in normal individuals, suggesting that the intake of $\beta$-cryptoxanthin-containing juice has an anabolic effect on bone mass in normal individuals.

Serum bone-specific alkaline phosphatase and $\gamma$-carboxylated osteocalcin are expressed in osteoblastic cells, ${ }^{19,20)}$ which stimulate bone formation. Serum $\gamma$-carboxylated osteocalcin concentration was found to be increased by the intake of the regular juice or the reinforced juice containing additional $\beta$ cryptoxanthin in normal individuals, although their intake did not cause a significant elevation of serum bone-specific alkaline phosphatase activity. This result suggests that the intake of $\beta$-cryptoxanthincontaining juice stimulates bone formation in normal individuals.

Serum bone TRAP is a specific marker enzyme in osteoclasts, ${ }^{17)}$ and the N-telopeptide of type I col- 
lagen is specifically formed following the stimulation of bone resorption. ${ }^{18)}$ The intake of $\beta$-cryptoxanthin reinforced juice for 28 and 56 days caused a significant decrease in serum bone TRAP activity and serum concentration of the $\mathrm{N}$-telopeptide of type I collagen in normal individuals. This result suggests that bone resorption is suppressed by the intake of $\beta$-cryptoxanthin-containing juice in normal individuals.

Serum calcium and inorganic phosphorus concentrations were observed to be decreased by the prolonged intake of $\beta$-cryptoxanthin-containing juice. This observation may result from the suppression of bone resorption caused by the intake of $\beta$ cryptoxanthin-containing juice.

The content of $\beta$-cryptoxanthin in the regular juice prepared from Satsuma mandarin was $802 \mu \mathrm{g}$ per $100 \mathrm{ml}$ of juice. This juice is supplied commercially. The intake of regular juice for 56 days caused a significant increase in serum $\gamma$-carboxylated osteocalcin concentration and a significant decrease in serum bone TRAP activity in normal individuals. Moreover, regular juice was supplemented with $\beta$ cryptoxanthin isolated from Satsuma mandarin; the content of $\beta$-cryptoxanthin was elevated to $1500 \mu \mathrm{g}$ per $100 \mathrm{ml}$ of juice. The intake of reinforced juice for 28 and 56 days caused a significant increase in serum $\gamma$-carboxylated osteocalcin concentration and a significant decrease in serum calcium concentration, serum bone TRAP activity, and serum Ntelopeptide of type I collagen in normal individuals. Thus, the reinforced $\beta$-cryptoxanthin juice had a potent effect on biochemical markers of bone metabolism. The intake of $\beta$-cryptoxanthin reinforced juice may be useful for bone health and the prevention of bone loss caused by increasing age.

The total intake of $\beta$-cryptoxanthin in normal individuals was $2880 \mu \mathrm{g}$ per day when the reinforced juice was given. If the body weight of normal individuals was about $55 \mathrm{~kg}$, the optimum dose of $\beta$ cryptoxanthin would be $52.4 \mu \mathrm{g}$ per kg body weight. The marked anabolic effect of $\beta$-cryptoxanthin has been shown to be induced by oral administration for 7 days with the dose of $100 \mu \mathrm{g} \beta$-cryptoxanthin $/ \mathrm{kg}$ body weight of rats. ${ }^{13)}$ It has been reported that the serum concentration of $\beta$-cryptoxanthin increases, due to the consumption of vegetable juice in women, to the range of $1.3 \times 10^{-7}$ to $5.3 \times 10^{-7} \mathrm{M}^{21)}$ The anabolic effect of $\beta$-cryptoxanthin on bone components was observed at $10^{-7}$ and $10^{-6} \mathrm{M}$ using rat femoral tissues in vitro. Presumably, the anabolic effect on bone metabolism with the intake of $\beta$-cryptoxan- thin reinforced juice results from a direct effect of $\beta$-cryptoxanthin on osteoblasts and osteoclasts in the bone tissues of normal individuals.

In conclusion, it has been shown that the intake of reinforced juice, which contains more $\beta$-cryptoxanthin than regular juice, may participate in bone health and the prevention of age-related bone loss.

\section{REFERENCES}

1) Bonjour, J.-P., Schurch, M.-A. and Rissori, R. (1996) Nutritional aspects of hip fractures. Bone, 18, 13951445.

2) Yamaguchi, M. and Gao, Y. H. (1998) Anabolic effect of genistein and genistin on bone metabolism in the femoral-metaphyseal tissues of elderly rats: The genistein effect is enhanced by zinc. Mol. Cell. Biochem., 178, 377-382.

3) Sugimoto, E. and Yamaguchi, M. (2000) Stimulatory effect of daidzein in osteoblastic MC3T3-E1 cells. Biochem. Pharmacol., 59, 471-475.

4) Yamaguchi, M. (2002) Isoflavone and bone metabolism: Its cellular mechanism and preventive role in bone loss. J. Health Sci., 48, 209-222.

5) Yamaguchi, M., Sugimoto, E. and Hachiya, S. (2001) Stimulatory effect of menaquinone-7 (vitamin $\mathrm{K}_{2}$ ) on osteoblastic bone formation in vitro. Mol. Cell. Biochem., 223, 131-137.

6) Yamaguchi, M., Kakuda, H., Gao, Y. H. and Tsukamoto, Y. (2000) Prolonged intake of fermented soybean (natto) diets containing vitamin $\mathrm{K}_{2}$ (manaquinone-7) prevents bone loss in ovariectomized rats. J. Bone Miner. Metab., 18, 71-76.

7) Tsukamoto, Y., Ichise, M. and Yamaguchi, M. (2000) Prolonged intake of dietary fermented soybean (natto) with the reinforced vitamin $\mathrm{K}_{2}$ (manaquinone-7) enhances circulating $\gamma$-carboxylated osteocalcin concentration in normal individuals. J. Health Sci., 46, 317-321.

8) Ono, R., Ma, Z. J. and Yamaguchi, M. (2000) Prolonged intake of fermented soybean diets with supplementation of isoflavone and saponin prevents bone loss in ovariectomized rats. J. Health Sci., 46, 70-74.

9) Yamaguchi, M., Taguchi, H., Gao, Y. H., Igarashgi, A. and Tsukamoto, Y. (1999) Effect of vitamin $\mathrm{K}_{2}$ (menaquinone-7) in fermented soybean (natto) on bone loss in ovariectomized rats. J. Bone Miner. Metab., 17, 23-29.

10) Yamacguhi, M. and Uchiyama, S. (2003) Effect of carotenoid on calcium content and alkaline phosphatase activity in rat femoral tissues in vitro: The unique anabolic effect of $\beta$-cryptoxanthin. Biol. 
Pharm. Bull., 26, 1188-1191.

11) Yamaguchi, M. and Uchiyama, S. (2004) $\beta$-Cryptoxanthin stimulates bone formation and inhibits bone resorption in tissue culture in vitro. Mol. Cell. Biochem., 258, 137-144.

12) Uchiyama, S. and Yamaguchi, M. (2004) Inhibitory effect of $\beta$-cryptoxanthin on osteoclast-like cell formation in mouse marrow cultures. Biochem. Pharmacol., 67, 1297-1305.

13) Uchiyama, S., Sumida, T. and Yamaguchi, M. (2004) Oral administration of $\beta$-cryptoxanthin induces anabolic effects on bone components in the femoral tissues of rats in vivo. Biol. Pharm. Bull., 27, 232235.

14) Uchiyama, S., Sumida, T. and Yamaguchi, M. (2004) Anabolic effect of $\beta$-cryptoxanthin on bone components in the femoral tissues of aged rats in vivo and in vitro. J. Health Sci., 50, 491-496.

15) Koyama, N., Ohara, K., Yokota, H., Kurome, T., Katayama, M., Hino, F., Kato, I. and Aki, T. (1991) A one step sandwich enzyme immunoassay for antibodies. J. Immunol. Methods, 139, 17-23.

16) Gomez, B., Jr., Ardakani, S., Ju, J., Jenkins, D., Cerelli, M. J., Daniloff, G. Y. and Kung, V. T. (1995) Monoclonal antibody assay for measuring bone-specific alkaline phosphatase activity in serum. Clin.
Chem., 41, 1560-1566.

17) Hallen, J. M., Alatalo, S. L., Suminen, H., Cheng, S., Janckila, A. J. and Vaananen, H. K. (2000) Tartrate-resistant acid phosphatase 5b: A novel serum marker of bone resorption. J. Bone Miner. Res., 15, 1337-1345.

18) Clemens, J. D., Herrick, M. V., Singer, F. R. and Eyre, D. R. (1997) Evidence that serum NTx (collagen-type I N-telopeptides) can act as an immunochemical marker of bone resorption. Clin. Chem., 43, 2058-2063.

19) Price, P. A. (1985) Vitamin K-dependent formation of bone gla protein (osteocalcin) and its function. Vitam. Horm., 42, 65-108.

20) Levy, J. R., Murray, E., Manolagass, S. and Olefsky, J. M. (1986) Demonstration of insulin receptors and modulation of alkaline phosphatase activity by insulin in rat osteoblastic cells. Endocrinology, 119, 1786-1792.

21) McEligot, A. J., Rock, C. L., Shanks, T. G., Flatt, A. W., Newman, V., Faerber, S. and Pierce, J. P. (1999) Comparison of serum carotenoid response between women consuming vegetable juice and woman consuming raw or cooked vegetable. Cancer Epidemiol. Biomarkers Prev., 8, 227-231. 